\title{
Barium Titanate and the Dielectric Response of Polystyrene-based Composites
}

\author{
O. Boorman, I.L. Hosier, M. Praeger, R. Torah, A.S. Vaughan, T Andritsch*, S.G Swingler, J. Topham, \\ Electronics and Computer Science, \\ University of Southampton, \\ Southampton SO17 1BJ, UK. \\ *E-mail: t.andritsch@soton.ac.uk
}

\begin{abstract}
Barium titanate $\left(\mathrm{BaTiO}_{3}\right)$ is characterized by real permittivity values as high as $\mathbf{1 0 , 0 0 0}$, depending upon structure. Consequently, in this work, this material was chosen to serve as a high permittivity filler for polystyrene (PS) based composites. In order to determine the influence of particle dimensions on the permittivity of the bulk material, the filler size was varied by means of ball milling, from the as-received microscopic filler size, to the mesoscopic and nanometric range. Filler size was measured by scanning electron microscopy (SEM). To explore the influence of fillgrade and particle size on the effective permittivity, a range of different composites was produced and subjected to dielectric spectroscopy. The results obtained were compared with effective medium theories. Since neither the Maxwell Garnet nor Bruggeman equations were able to reproduce the dependency of permittivity on composition seen experimentally, the Lichtenecker Rother equation was chosen and found to provide a good fit for the experimental data.
\end{abstract}

\section{INTRODUCTION}

Composite materials are often produced by adding an inorganic filler to a polymer, in order to modify some macroscopic physical property of the system. For example, the inclusion of inorganic filers can improve thermal conductivity or may be used to match the thermal expansion coefficient of the insulation and conductor in a bushing. However, while the addition of a large volume fraction of an inorganic material may well bring desired thermal benefits as illustrated above, it will also affect mechanical and electrical characteristics, potentially, in unwanted ways. For example, composites containing filler particles of the order of micrometres in size generally exhibit breakdown strengths that are significantly reduced compared with the unfilled polymer.

The real part of the permittivity of a material is an important parameter, which represents the energy stored within the dielectric. Consequently, many approaches have been devised in order to determine the effective permittivity of a binary composite based upon an array of inclusions within a continuous matrix. Relationships such as the Lichtenecker Rother, Maxwell Garnett and Bruggeman effective medium equations $[1,2]$ consider the real part of the permittivity of the composite system, $\varepsilon_{c}{ }^{\prime}$, in terms of the volume fraction of the particulate phase, $\phi_{p}$, the permittivity of the particles, $\varepsilon_{p}{ }^{\prime}$, and the permittivity of the matrix $\varepsilon_{m}{ }^{\prime}$. Although modified formulations have been developed to account for the shape of the inclusions, in general, the size of the inclusions is not considered.
Recent studies of nanocomposites have shown unexpected effects, in which the permittivity of systems containing a low volume fraction of nanofiller have been observed to fall outside the absolute bounds; that is outside the limits set by the permittivities of the two components. This has been interpreted in terms of the dominant role of interfaces in such systems, whereupon, effective medium theories need explicitly to consider particles, matrix and interphase components.

In this study, we set out to examine particle size effects in composite materials where the constituents were chosen deliberately to be characterised by a high contrast in relative permittivity between the two phases. This choice should manifest itself in extreme behaviour and, thereby, both provide a rigorous test of effective medium theories and magnify any particle size effects.

\section{EXPERIMENTAL}

\section{A. Materials}

The materials used in this work were produced using barium titanate $\left(\mathrm{BaTiO}_{3}\right)$ and atactic polystyrene (PS), both obtained from Sigma Aldrich. The $\mathrm{BaTiO}_{3}$ was characterised by a mean particle size smaller than $2 \mu \mathrm{m}$ and the PS by an average molar mass of $192.000 \mathrm{~g} \cdot \mathrm{mol}^{-1}$, according to the suppliers.

The $\mathrm{BaTiO}_{3}$ was used as supplied and was also subjected to ball milling using a Netszch Microcer bead mill. The $\mathrm{BaTiO}_{3}$ powder was mixed in a $200 \mathrm{ml}$ water suspension with a BYK dispersant. This mix was subsequently circulated through the ball mill loaded with $50 \mathrm{ml}$ of $0.4-0.7 \mathrm{~mm}$ Yttria beads and a $0.1 \mathrm{~mm}$ filter. The milling speed was set to $2500 \mathrm{rpm}$ and two different circulation times were used: $30 \mathrm{~min} ; 24 \mathrm{~h}$.

Composites were generated from the above materials using a standard solution mixing route. In this, the PS was initially dissolved in dichloromethane (DCM), with stirring, to give a solution corresponding to a weight/volume concentration of $\sim 10 \%$. The required mass of filler was added to this solution, dispersed with further stirring as well as sonication and, finally, the DCM containing both the polymer and filler was added to the non-solvent methanol. This induces rapid precipitation of the polymer and entrains the inorganic filler within the resulting polymeric gel. The resulting composite was initially dried to remove the bulk of the solvent, before being melt pressed a number of times to remove any final traces of methanol and DCM. Finally, specimens, nominally 
$\sim 200 \mu \mathrm{m}$ in thickness, were produced for dielectric spectroscopy using a Specac press fitted with a precision thin film attachment.

\section{B. Material Characterization}

The actual composition of the composite systems prepared as above was determined by thermogravimetric analysis (TGA), use a Perkin Elmer Pyris 1 system. The experimental protocol involved heating the specimen (about $5 \mathrm{mg}$ in mass) from $50{ }^{\circ} \mathrm{C}$ to $650{ }^{\circ} \mathrm{C}$ at a rate of $10{ }^{\circ} \mathrm{C} / \mathrm{min}$, under oxidizing conditions, to remove the organic compound and leave the filler material as remaining mass.

Typical composite specimens, all containing a nominal $50 \%$ by mass of $\mathrm{BaTiO}_{3}$, were examined by scanning electron microscopy (SEM), to explore both the structure of the filler and its dispersion within the matrix. These samples were etched using a $1 \%$ solution of potassium permanganate in an acidic mix composed of 5 parts concentrated sulphuric acid to 2 parts orthophosphoric acid to 2 parts water and then recovered following prescribed procedures [3].

Thin film specimens for dielectric spectroscopy were first gold coated on either side, to ensure good contact with the electrodes of the sample cell. Spectra were then obtained at room temperature from $1 \mathrm{~Hz}$ to $50 \mathrm{kHz}$ using a Solartron 1260A impedance analyser fitted with a Solartron 1296 dielectric interface.

\section{RESULTS}

\section{A. TGA Analysis and Composite Composition}

A typical TGA trace obtained from a PS sample with 50 wt.\% of $\mathrm{BaTiO}_{3}$ milled for 30 minutes is shown in Fig.1. From this, it is evident that the primary decomposition of the organic components occurs over the temperature range 350$475^{\circ} \mathrm{C}$. Thereafter, the stable mass of the residue suggests that little in the way of carbon residue is formed during the primary decomposition. These decomposition characteristics are consistent with the behaviour of a polymer such as PS, suggesting that the residue is indeed related to the initial organic fraction within the system. The resulting compositional data derived from all the $\mathrm{BaTiO}_{3}$-based composites are shown in Table 1, from which it is evident that the nominal composition, based on the masses of the components used to formulate the composite, correlates reasonably well with the derived TGA data for most filler loadings. For low filler content we see a rather large deviation for low filler contents of ball milled composites however.

Table 1: Composition data for all the composites considered here

\begin{tabular}{c|c|c}
\hline $\begin{array}{c}\text { Nominal Filler } \\
\text { Loading }\end{array}$ & $\begin{array}{c}\text { Particle } \\
\text { preparation }\end{array}$ & TGA Filler Loading \\
\hline $10 \%$ & As received & $8.9 \%$ \\
$20 \%$ & As received & $20.2 \%$ \\
$50 \%$ & As received & $44.2 \%$ \\
$75 \%$ & As received & $76.8 \%$ \\
$10 \%$ & Milled 30 min & $15.2 \%$ \\
$20 \%$ & Milled 30 min & $14.5 \%$ \\
$50 \%$ & Milled 30 min & $42.4 \%$ \\
$75 \%$ & Milled 30 min & $68.1 \%$ \\
\hline
\end{tabular}

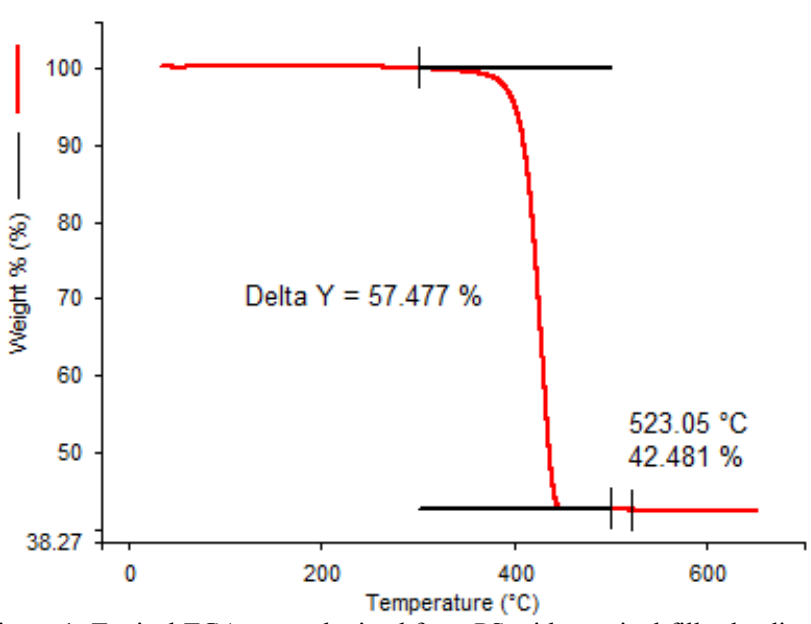

Figure 1: Typical TGA trace obtained from PS with nominal filler loading of 50 wt. $\%$ of $\mathrm{BaTiO}_{3}$. Actual filler loading differed from nominal filler loading, for list of actual filler loadings see Table 1.

\section{B. Composite Structure}

Fig. 2 shows a range of SEM micrographs obtained from PS samples with a nominal filler loading of $50 \%$ by weight. The filler in Fig. 2 has not been processed by means of ball milling and is therefore referred to as non-milled. It can be seen in Fig. $2 \mathrm{a}$ that there is an even, random dispersion of $\mathrm{BaTiO}_{3}$ across the entire sample. However, looking closer, Fig. $2 b$ shows that non-milled samples contain a number of agglomerations. Conventional physical mixing methods are not enough to break up the agglomerates [4].

Fig. 3 shows SEM micrographs for PS samples with the same nominal filler loading. However, the $\mathrm{BaTiO}_{3}$ has been ball milled for 30 minutes, which has effectively broken up the agglomerates, as seen in Fig. 3b. Such micrographs clearly show that this method has been successful. Instead of agglomerations of primary particles, we can observe that we have both a good dispersion of barrel-shaped primary particles, as well as a good distribution of those particles across the composite (Fig. 3a).

The distribution of particles after 24 hours of ball milling can be seen in Fig. 4a. Compared with Fig. 3a it is apparent, that the distribution of particles has become worse. In fact, the dispersion is arguably worse than for as-received samples shown in Fig. 2a. At higher magnification (see Fig. 4b), it can be seen that the primary $\mathrm{BaTiO}_{3}$ particles of approximately $500 \mathrm{~nm}$ (Fig. 3b) are broken up into smaller fragments, which in turn form larger agglomerates. The formation of agglomerates then leads to a worse dispersion throughout the composite.

\section{Permittivity of $\mathrm{BaTiO}_{3}$-based Composites}

The permittivity of samples is shown as a function of fillgrade in Fig. 5. The sample weight percentages have been adjusted according to TGA results in Table I and converted into volume fractions according to densities given by the datasheets provided by the manufacturer. The Lichtenecker- 

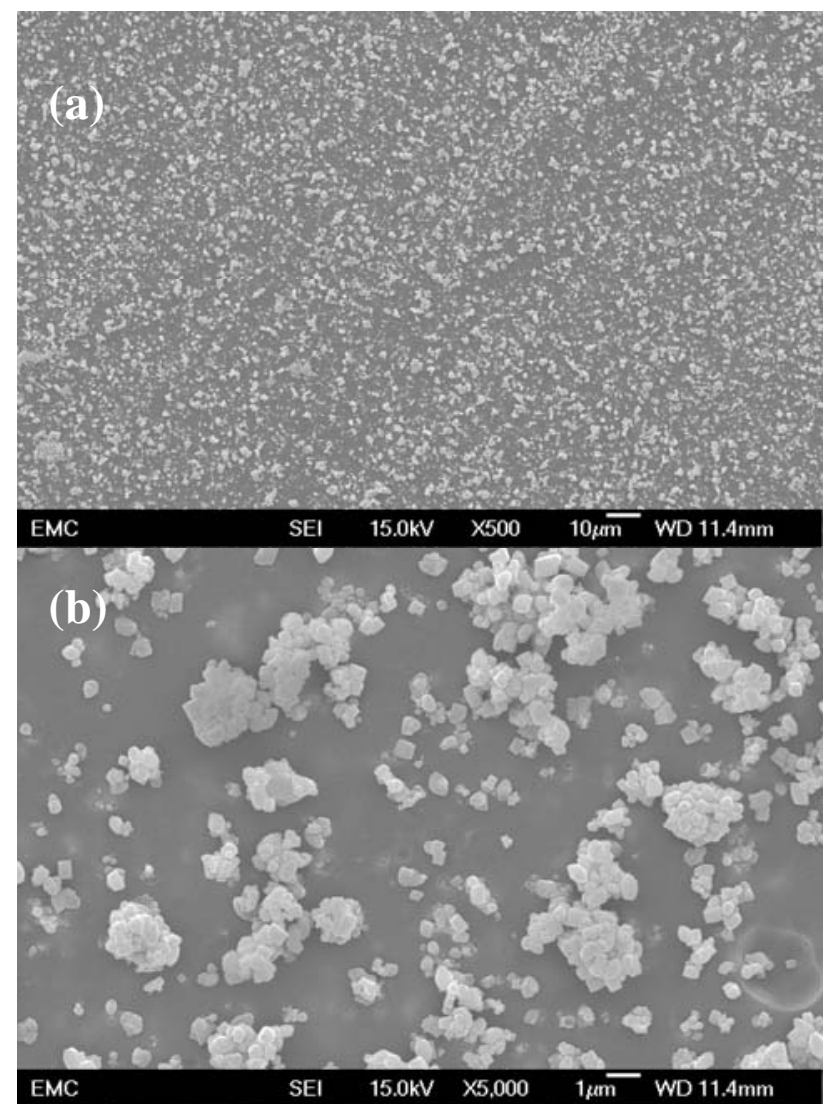

Figure 2: SEM micrographs of PS with a nominal filler loading of $50 \mathrm{wt} . \%$ of non milled $\mathrm{BaTiO}_{3}$; (a) distribution of agglomerates; (b) dispersion state of the particles.

Rother equation (1) has been used to fit the data, since the Lichtenecker-Rother fits data better than e.g. Bruggeman [5].

$$
\log \varepsilon_{c}{ }^{\prime}=\phi_{p} \log \varepsilon_{p}{ }^{\prime}+\left(1-\phi_{p}\right) \log \varepsilon_{m}{ }^{\prime}
$$

With the parameter $\varepsilon_{\mathrm{m}}$ fixed at the value of 2.75 for PS, the parameter $\varepsilon_{\mathrm{p}}$ could be calculated to be about $524 \pm 72$ for asreceived $\mathrm{BaTiO}_{3}$, and $569 \pm 83$ for samples that were ball milled for 30 minutes. These values are at the bottom end for what we would expect from $\mathrm{BaTiO} 3$, but fall within the expected range of values for small filler sizes [6].

As it can be seen from Fig. 5, the trend for both non-milled and ball milled samples is very similar, namely a monotonic increase of the real permittivity with filler volume fraction. There are only minor variations in the fitting parameters, which are well within the confidence bounds.

\section{DISCUSSION}

The SEM micrographs show that ball milling is a viable way of breaking up agglomerates of $\mathrm{BaTiO}_{3}$ particles. However, if used for prolonged periods of time, the process breaks up the primary particles of $\mathrm{BaTiO}_{3}$ and results in re-agglomeration, where the resulting small fragments of barium titanate act as glue that bind together the remaining larger particles. In the course of this work, two milling times were chosen: 30 minutes and 24 hours; 30 minutes seems to deliver very good

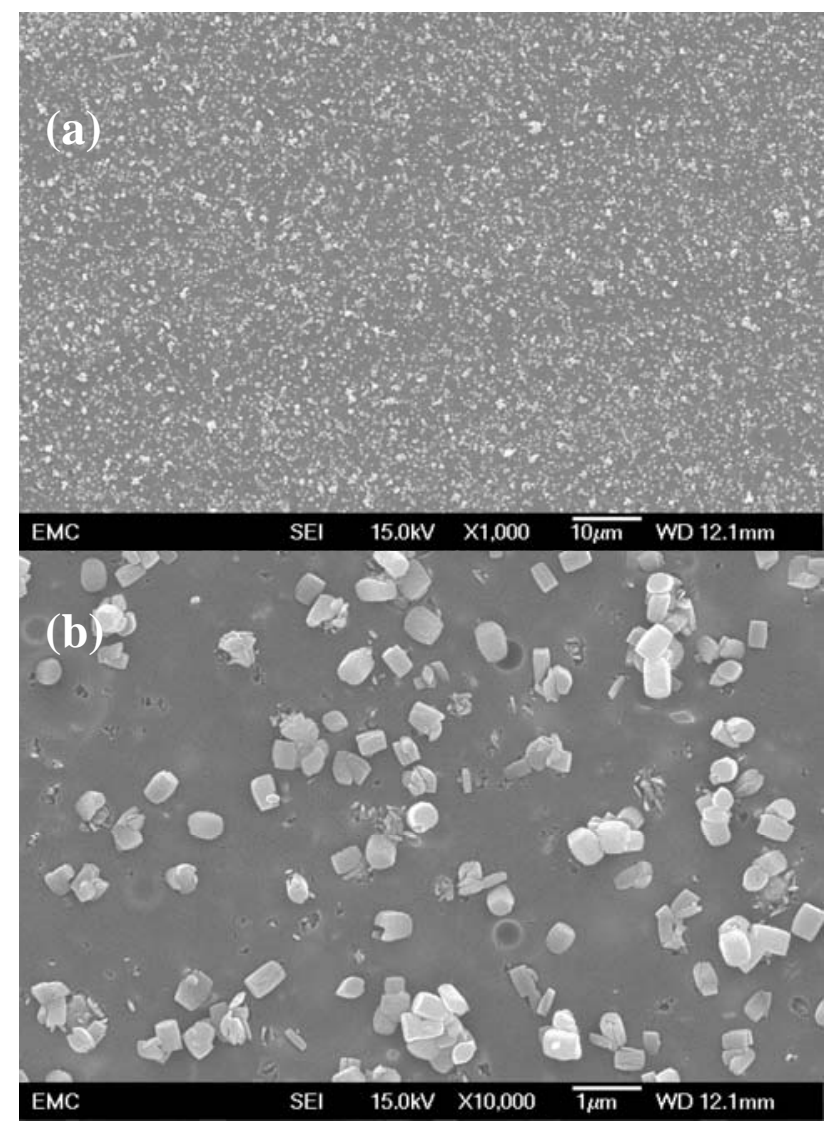

Figure 3: SEM micrographs of PS with a nominal filler loading of $50 \mathrm{wt} . \%$ of $\mathrm{BaTiO}_{3}$ after 30 minutes of ball milling; (a) distribution of agglomerates; (b) dispersion state of the particles.

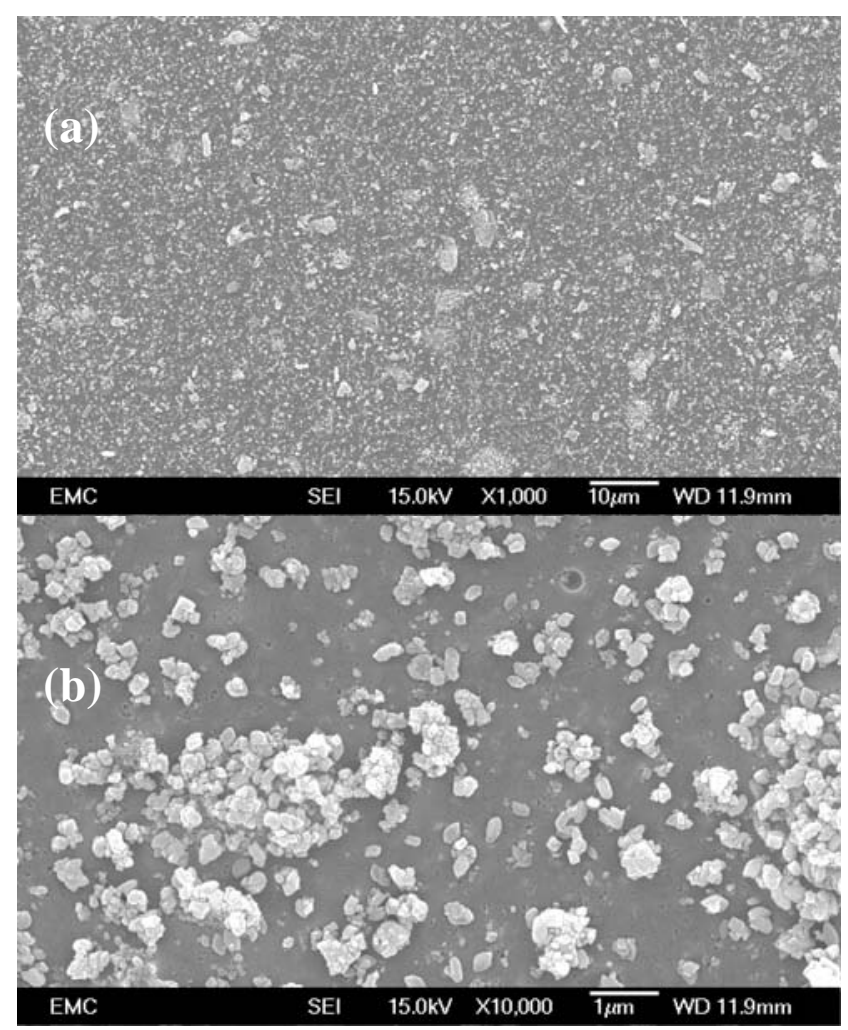

Figure 4: SEM micrographs of $\mathrm{PS} / \mathrm{BaTiO}_{3} 50 \%$ after 24 hours of ball milling; (a) distribution of agglomerates; (b) dispersion state of the particles. 


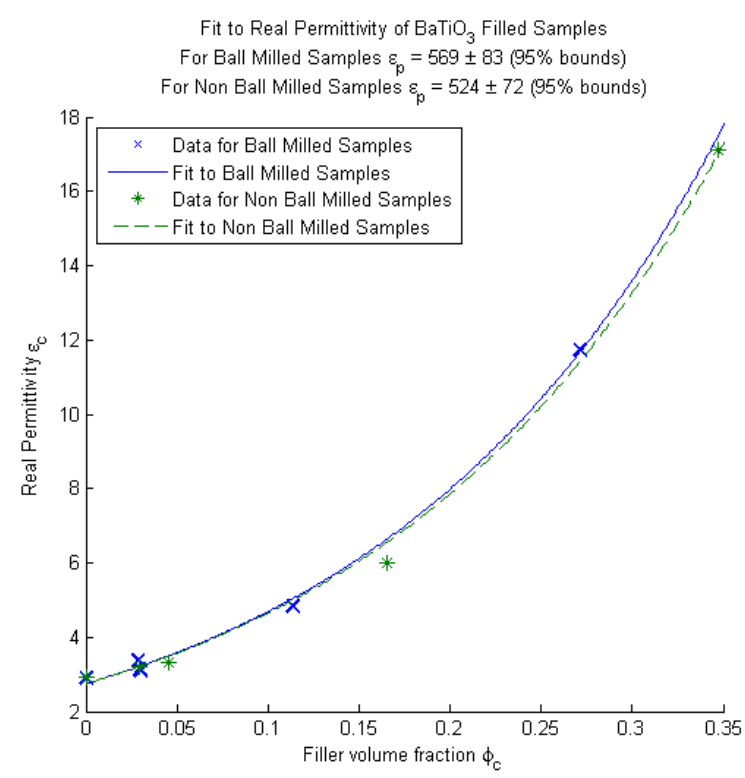

Figure 5: Data from dielectric spectroscopy showing the real permittivity of BaTiO3 filled polystyrene samples as a function of filler volume fraction. It can be seen that the Lichtenecker Rother fit does not differ significantly for both types of fillers (ball milled, non ball milled).

results in terms of particle distribution and dispersion throughout the composite. However, as seen in Fig. 3b, even after 30 minutes there are a number of fractured primary particles, while small clumps remain. Using ball milling for 24 hours is causing re-agglomeration of previously dispersed particles, although this re-agglomeration could possibly be prevented by adjusting the $\mathrm{pH}$ of the system. However, it has been shown that the isoelectric point (IEP) $\mathrm{pH}$ of stoichiometric $\mathrm{BaTiO}_{3}$ can vary widely, between $\mathrm{pH} 4$ to above pH 11 and chemical instability is an issue with $\mathrm{BaTiO}_{3}$ [7]. With such a wide range of IEP $\mathrm{pH}$ depending on various factors, including particle size, composition and structure, creating a stable colloidal dispersion prior to mixing with the polymer is rather challenging. More work is needed to characterize the impact of ball milling on the IEP $\mathrm{pH}$ and hence measures to counter the re-agglomeration effects witnessed in the SEM micrographs.

In terms of dielectric behavior, it is interesting to see how the composites can be described according to the Lichtenecker Rother equation over a wide range of filler volume fractions, even though the assumed real permittivity of the particles is on the lower end of expected values. But since it seems consistent for all observed dispersion states, there is no reason to assume that polymer interactions at the interface have significant impact on the composite permittivity. Unlike nanoparticles, where the interfacial volume seems to be large enough to result in significant changes to the composite permittivity, the filler volume seems to be the main parameter determining the real permittivity in composites with fillers in the micro-scale. The dispersion state has no observable effect, hence agglomerations of smaller particles are interchangeable with larger particles of the same volume in terms of the dielectric response.

\section{CONCLUSIONS}

Ball milling for 30 minutes has been used to great effect in combination with a solvent blending process to achieve a good dispersion of $\mathrm{BaTiO}_{3}$ particles $(\sim 500 \mathrm{~nm}$ in size $)$ in polystyrene. While increasing the time for ball milling to 24 hours reduced the average particle size by breaking up the primary particles, this did not result in a good dispersion of particles by physical mixing methods. Changes to the $\mathrm{BaTiO}_{3}$ structure due to ball milling and a subsequent shift of the isoelectric $\mathrm{pH}$ are likely causes of the the re-agglomeration of particles, compared to other composite systems.

The real part of the complex permittivity was measured over a wide range of filler fractions up to $35 \%$ by volume. It has been shown that the agglomeration state of microscale particles does not change the permittivity significantly. Hence, agglomerations of smaller particles can be converted into larger particles with few adjustments for the purpose of modelling the permittivity.

\section{ACKNOWLEDGMENT}

The authors gratefully acknowledge the RCUK's Energy Programme for the financial support of this work through the Top \& Tail Transformation programme grant, EP/I031707/1.

\section{REFERENCES}

[1] V. Myroshnychenko and C. Brosseau, "Finite-element modeling method for the prediction of the complex effective permittivity of two-phase random statistically isotropic heterostructures", J. Appl. Phys., vol. 97, 044101, 2005.

[2] M. Kühn and H. A. Kliem, "A numerical method for the calculation of dielectric nanocomposites", IEEE Trans. Diel. Electr. Insul., vol. 17, pp. 1499-1508, 2010.

[3] R. H. Olley and D. C. Bassett, "An improved permanganic etchant for polyolefins," Polymer, vol. 23, pp. 1707-1710, 1982.

[4] T. Andritsch, D. Fabiani, I. Ramirez, "Nanodielectrics: Examples for Preparation and Microstructure", IEEE Electrical Insulation Magazine, vol. 29, Issue 6, pp. 21-28, 2013.

[5] J. Topham et al. "Dielectric Studies of PS-based Composite Systems", Procedings of the annual CEIDP, Des Moines, Iowa, 2014.

[6] H. Ravindra et al. "Characterization, dielectric and electrical behaviour of $\mathrm{BaTiO} 3$ nanoparticles prepared via titanium(IV) triethanolaminato isopropoxide and hydrated barium hydroxide", Bull. Mater. Sci, vol. 37, pp. 481-489, 2014.

[7] U. Paik and V.A. Hackley, "Influence of Solids Concentration on the Isoelectric Point of Aquaeous Barium Titanate", J. Am. Ceram. Soc. Vol. 83, No. 10, pp. 2381-2384, 2000. 